\title{
Ambient-pressure synthesis of single-crystal $\mathrm{MgB}_{2}$ and their superconducting anisotropy
}

\author{
Y. Machida* and S. Sasaki \\ Materials and Structures Laboratory, Tokyo Institute of Technology, 4259 Nagatsuta, Midori-ku, Yokohama 226-8503, Japan \\ H. Fujii \\ National Institute for Materials Science, 1-2-1, Sengen, Tsukuba 305-0047, Japan
}

M. Furuyama, I. Kakeya, and K. Kadowaki

Institute of Materials Science, University of Tsukuba, 1-1-1, Tennoudai, Tsukuba 305-8573, Japan

(Received 27 July 2002; revised manuscript received 15 November 2002; published 18 March 2003)

\begin{abstract}
We synthesized single crystalline $\mathrm{MgB}_{2}$ under ambient pressure by using conventional materials and equipment. The single crystals of $\mathrm{MgB}_{2}$ were of good quality, where the crystal structure refinements were successfully converged with $R=0.020$. The measurements of the magnetic properties yielded a sharp superconducting transition at $38 \mathrm{~K}$ with transition width $\Delta T_{c}=0.8 \mathrm{~K}$. The upper critical field for applied field parallel to the $a b$ plane $\left(H_{c 2}^{a b}\right)$ reveals a positive curvature, while $H_{c 2}$ parallel to the $c$ axis $\left(H_{c 2}^{c}\right)$ increases linearly in temperature dependence, which yields a temperature dependence of the superconducting anisotropy ratio of $\gamma$ $=H_{c 2}^{a b} / H_{c 2}^{c}$ with $\gamma \sim 1$ near $T_{c}$ and 4.0 at $25 \mathrm{~K}$.
\end{abstract}

DOI: 10.1103/PhysRevB.67.094507

PACS number(s): 74.25.Bt, 74.25.Ha, 74.62.Bf, 74.25.Op

\section{INTRODUCTION}

The recent discovery of superconductivity at $39 \mathrm{~K}$ in magnesium diboride $\mathrm{MgB}_{2}$ (Ref. 1) has attracted great scientific interest because of the highest $T_{c}$ among conventional metals and intermetallic compounds. Several experiments indicated phonon-mediated $s$-wave BCS superconductivity 2,3 and the appearance of a double energy gap was predicted. ${ }^{4,5}$ Specific heat ${ }^{6}$ and spectroscopic ${ }^{7}$ measurements, scanning tunneling spectroscopy, ${ }^{8,9}$ gave evidence of this prediction. However, several key parameters such as the upper critical fields $H_{c 2}$ and their anisotropy ratio $\gamma$, the magnetic penetration depth $\lambda$, the coherence length $\xi$ and Ginzburg-Landau parameter $\kappa$ are not well established because of the difficulty of growing high quality $\mathrm{MgB}_{2}$ single crystals.

In particular, the anisotropy ratio $\gamma=H_{c 2}^{a b} / H_{c 2}^{c}$ is important to characterize superconducting properties and for applications of $\mathrm{MgB}_{2}$. Here $H_{c 2}^{a b}$ and $H_{c 2}^{c}$ are the in-plane and out-of-plane upper critical fields, respectively. The reported $\gamma$ values vary widely depending on the measurement methods or on the sample types. The values determined from resistivity on polycrystals,${ }^{10}$ aligned crystallites,${ }^{11} c$-axis oriented films, ${ }^{12-14}$ and single crystals ${ }^{15-23}$ have been reported to be $6-9,1.7,1.3-2$, and $2.6-3$, respectively.

So far, there have been several reports on superconducting properties in $\mathrm{MgB}_{2}$ single crystals. ${ }^{15,17,18,24}$ Most of these crystals were synthesized under high pressure, typically 5 $\mathrm{GPa}$, with high pressure facilities. Shapes of crystals grown under high pressure are mostly irregular, so that it is almost impossible to recognize the correspodence between the crystal shapes and crystalographic axes. This causes experimental difficulties for measurements on anisotropic properties.

In this paper, we report on a method to grow a single crystal under ambient pressure, which does not require special equipments. Then we characterize the crystals in various ways. The grown crystals shows good crystalographic quali- ties compared with crystals grown under high pressure in the literature, ${ }^{15,24}$ and the correspondence between sample edges and crystalographic axis is not hard to recognize. Using these crystals, we investigated the superconducting properties through magnetization measurements, and found that the anisotropy parameter $\gamma$ of $H_{c 2}$ strongly depends on temperature, in particular just below $T_{c}$.

\section{AMBIENT PRESSURE SYNTHESIS AND CHARACTERIZATION}

The single crystals were grown in the stainless (SUS304) tube. Typical dimensions of stainless tubes are 32, 1.5, and $110 \mathrm{~mm}$ for the outer diameter, the wall thickness, and the length, respectively. The inner surface of the tube was sealed by Mo sheet $(99.95 \%$, Nilaco Co.) with the size of 0.05

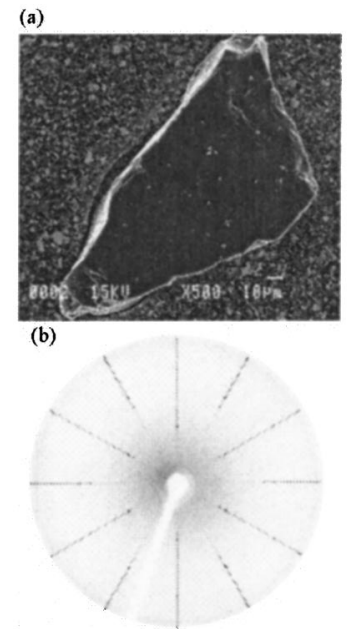

FIG. 1. (a) SEM image of a $\mathrm{MgB}_{2}$ single crystal with a size of about $100 \mu \mathrm{m}$. (b) X-ray precession photograph of the single crystal. 
TABLE I. Structural parameters of single crystals. Lattice constants: $a=b=3.0863(4) \AA, c=3.5178(4) \AA$. Agreement factors: $R=0.020, R_{w}=0.027$ ( ${ }_{w}=$ weight).

\begin{tabular}{lcccc}
\hline \hline Atom & atomic orientation & $U_{11}\left(\AA^{2}\right)$ & $U_{33}\left(\AA^{2}\right)$ & $B_{e q}\left(\AA^{2}\right)$ \\
\hline $\mathrm{Mg}$ & $(0,0,0)$ & $0.0078(2)$ & $0.0054(2)$ & $0.382(2)$ \\
$\mathrm{B}$ & $(1 / 3,2 / 3,1 / 2)$ & $0.0068(2)$ & $0.0058(2)$ & $0.371(2)$ \\
\hline
\end{tabular}

$\times 100 \times 200 \mathrm{~mm}^{3}$ to avoid direct reaction between the container materials and $\mathrm{Mg} / \mathrm{B}$ materials. One end of the SUS304 tube was pressed with a vise and sealed in an Ar gas atmosphere by arc welding. The starting materials of a B $(99.9 \%$, Furuuchi Chemical Co.) chunk with the size of $3 \sim 5 \mathrm{~mm}$ and a Mg (99.99\%, Furuya Metal Co.) chunk, which was cut out with the size of about $1 \mathrm{~cm}^{3}$ from $\mathrm{Mg}$ block, were filled inside the tube. Then the other end of the tube was pressed with a vise and sealed in an Ar gas atmosphere by arc welding, as well. For crystal growth, the temperature of the furnace inside, which the cruscible was put, was raised from room temperature to $1200{ }^{\circ} \mathrm{C}$ for $40 \mathrm{~min}$. The single crystals finally obtained were about $100-300 \mu \mathrm{m}$, which had a partly hexagonal shape with a shiny golden color when observed under an optical microscope.

The single crystal images observed by a scanning electron microscope (SEM) is shown in Fig. 1(a). The crystals were found to have very flat surfaces. The structural analysis was carried out using a x-ray precession camera, a four-circle diffractometer, and a transmission electron microscope (TEM). The X-ray precession photograph indicated that the crystal has a hexagonal structure, as shown in Fig. 1(b). The diffraction data were collected by using graphite monochromated $\mathrm{Mo} K_{\alpha}$ radiation at room temperature, and refined by the least-square procedure using 86 reflections as the average of the measured 866 reflections. Finally, we obtained structural the parameters as shown in Table I.

To confirm the structure of the $\mathrm{MgB}_{2}$ phase, we took a

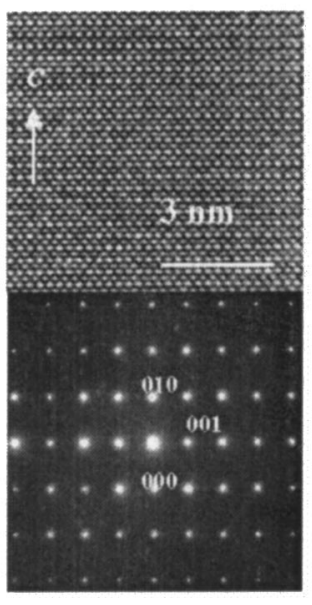

$<110>$

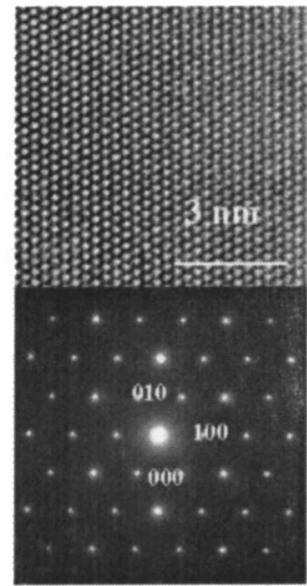

$<001>$
FIG. 2. Electron diffraction patterns and HRTEM images of a $\mathrm{MgB}_{2}$ single crystal for beam directions of [110] and [001] in the hexagonal structure.

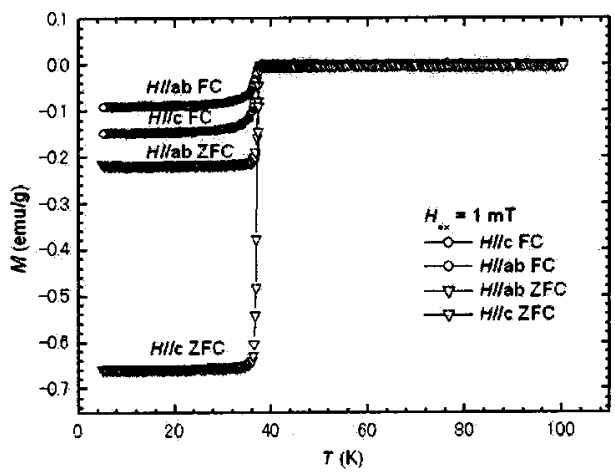

FIG. 3. Temperature dependent magnetization curves for the $\mathrm{MgB}_{2}$ single crystal. FC and $\mathrm{ZFC}$ denote the field cooling and zero field cooling curves, respectively.

plane view of the high resolution transmission electron microscope (HRTEM) images and electron diffraction patterns in selected areas for beam directions of [110] and [001], as shown in Fig. 2, which indicated an atomic arrangement with the $P 6 / \mathrm{mmm}$ cell of $\mathrm{MgB}_{2}$. From the left hand side of Fig. 2 , we recognize two kind of layers with different spot sizes alternately stacking along the $c$ axis. Neither extra spots nor streaks were found, indicating that the crystal is of high quality.

\section{ANISOTROPY OF THE UPPER CRITICAL FIELD}

The temperature dependence of the magnetization curve was measured at $1 \mathrm{mT}$ along the $c$ axis and the $a b$ plane by a superconducting quantum interference device magnetometer. Figure 3 represent the results of magnetization measurements for the $\mathrm{MgB}_{2}$ single crystal. It shows the $M(T)$ curves in the zero-field-cooling and field-cooling modes. The onset of superconducting transition was obtained at $T_{c}=38 \mathrm{~K}$, with transition widths $\Delta T_{c}=0.8 \mathrm{~K}$ both for $H \| a b$ and for $H \| c$, indicating the high quality of the samples, where $\| a b(\| c)$ denotes the field $H$ perpendicular (parallel) to the $c$ axis, respectively. The transition temperatures are slightly lower than $T_{c}$ of polycrystalline speciments $(\simeq 39 \mathrm{~K})$, as well as those of single crystals described in previous results. ${ }^{15,17,18,24}$ The suppression of $T_{c}$ was considered to be due to impurity contamination from container materials $(\mathrm{BN}$, $\mathrm{Mo}$, and $\mathrm{Nb}$ ). In order to check the contamination, we per-

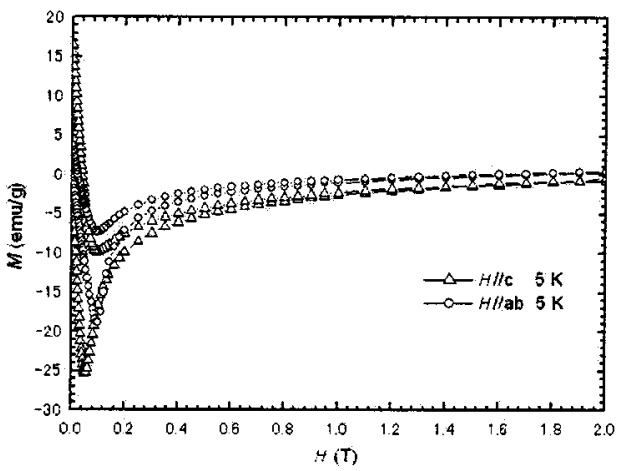

FIG. 4. The magnetic hysteresis curves $M(H)$ at $5 \mathrm{~K}$. 


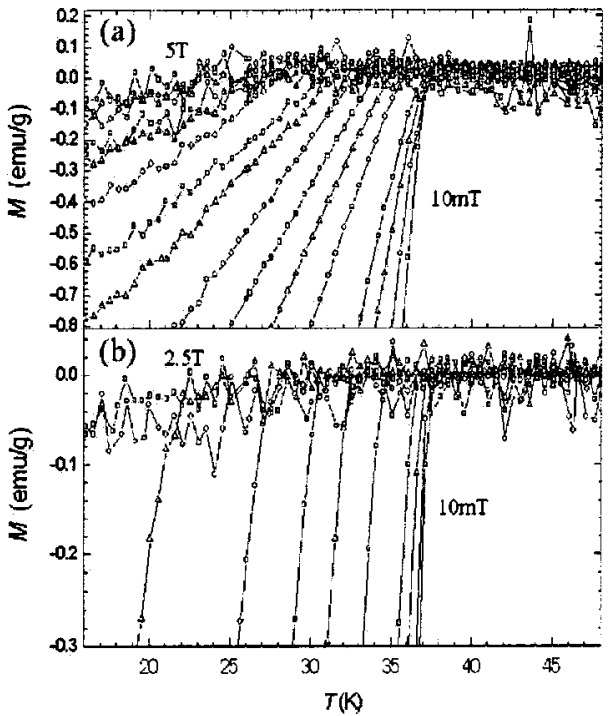

FIG. 5. Temperature dependence of the magnetization on zerofield cooling in several fields for (a) $H \| a b$ and (b) $H \| c$.

formed a qualitative analysis by an electron probe microanalyzer (EPMA). As a result, no contamination from our container elements (Mo, Fe, Ni, and $\mathrm{Co}$ ) was detected within a $0.1 \%$ accuracy.

Figure 4 shows the magnetic hysteresis curves $M(H)$ at 5 $\mathrm{K}$ for applied fields up to $2 \mathrm{~T}$ for $H \| a b$ and for $H \|_{c}$, indicating the characteristic curve of type-II superconductors with very small hysteresis loops. This implies that the single crystal has slight pinning effects, indicating pure single crystals with high quality.

Figure 5 shows the temperature dependence of magnetization $M(T)$ curves on warming after field cooling of the sample for (a) $H \| a b$ in a magnetic field up to $5 \mathrm{~T}$ and (b) $H \| c$ in a magnetic field up to $2.5 \mathrm{~T}$. The superconducting transition shifts to lower temperatures as the field is increased. The superconducting transition temperatures in finite fields are determined by extrapolating the negative part of the $M(T)$ curve linearly and by finding the crossing point to the horizontal line extended from the normal state. Using this criterion, the upper critical fields of $\mathrm{MgB}_{2}$ for applied fields $H \| a b$ and $H \| c$ are obtained, as shown in Fig. 6(a). It
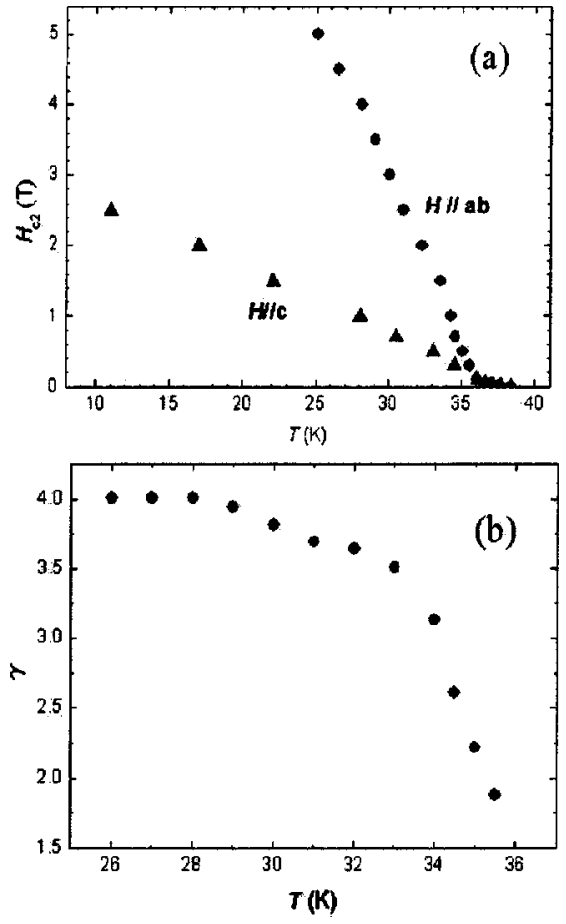

FIG. 6. (a) Upper critical field for $H_{c 2}^{a b}$ and $H_{c 2}^{c}$ determined from the onset of the superconductivity in Figs. 5(a) and 5(b) as a function of the temperature. $H_{c 2}^{a b}$ reveals a positive curvature, while $H_{c 2}^{c}$ has a linear temperature dependence. (b) Temperature dependence of the upper critical field anisotropy $\gamma=H_{c 2}^{a b} / H_{c 2}^{c}$.

is seen that $H_{c 2}^{a b}$ shows a positive curvature in the temperature dependence near $T_{c}$, and then rises rapidly at lower temperatures. In contrast, $H_{c 2}^{c}$ increases linearly with decreasing temperature. These temperature dependences of $H_{c 2}$ have also been observed in $\mathrm{MgB}_{2}$ single crystals by Lyard et $a .^{23}$ and were thought to be a characteristic feature of layered superconductors such as $\mathrm{NbSe}_{2} \cdot{ }^{26}$ Therefore, the anisotropy ratio $\gamma=H_{c 2}^{a b} / H_{c 2}^{c}$ is found to be temperature dependent, as displayed in Fig. 6(b). It increases from about 1 near $T_{c}$ to 4.0 at $25 \mathrm{~K}$. The extrapolation of $H_{c 2}^{a b}$ and $H_{c 2}^{c}$ lines to the zero temperature axis yields $H_{c 2}^{a b}(0) \sim 13.6 \mathrm{~T}$ and $H_{c 2}^{c}(0) \sim 3.4 \mathrm{~T}$, with $\gamma \sim 4.0$. Using the Ginzburg-Landau equation $H_{c 2}=\Phi_{0} /\left(2 \pi \xi^{2}\right)$, the coherence lengths $\xi$ were

TABLE II. Comparison of physical parameters with single crystals prepared by different methods.

\begin{tabular}{|c|c|c|c|c|c|}
\hline Parameter & Our sample & $\begin{array}{c}\text { References } \\
21,19 \text {, and } 24\end{array}$ & $\begin{array}{l}\text { References } \\
15 \text { and } 25\end{array}$ & $\begin{array}{l}\text { M. Xu et al. } \\
\quad \text { (Ref. 18) }\end{array}$ & $\begin{array}{c}\text { References } \\
17,20 \text {, and } 23\end{array}$ \\
\hline$a(\AA)$ & $3.0863(4)$ & $3.085(1)$ & $3.0851(5)$ & $3.047(1)$ & $3.09 \pm 0.06$ \\
\hline$c(\AA)$ & $3.5178(4)$ & $3.518(2)$ & $3.5201(5)$ & $3.404(1)$ & \\
\hline$R$ & 0.020 & $0.015-0.020$ & 0.018 & & \\
\hline$R_{w}$ & 0.027 & $0.015-0.020$ & 0.025 & & \\
\hline$T_{c}(\mathrm{~K})$ & 38 & $38-39$ & $38.1-38.3$ & 39 & 38 \\
\hline$H_{c 2}^{a b}(0)(\mathrm{T})$ & 13.6 & $14.5,^{\mathrm{a}} 23^{\mathrm{b}}$ & $21-22$ & 19.8 & 17 \\
\hline$H_{c 2}^{c}(0)(\mathrm{T})$ & 3.4 & $3.18,{ }^{\mathrm{a}} 3.1^{\mathrm{b}}$ & $7.0-7.5$ & 7.7 & 3.5 \\
\hline$\gamma(\mathrm{T})$ & $1\left(T_{c}\right)-4.0(25 \mathrm{~K})$ & $1\left(T_{c}\right)-4.2(22 \mathrm{~K}),{ }^{\mathrm{a}} 2.8(35 \mathrm{~K})-6(15 \mathrm{~K})^{\mathrm{b}}$ & $2.2\left(T_{c}\right)-3(30 \mathrm{~K})$ & $2.6(0 \mathrm{~K})$ & $2\left(T_{c}\right)-4.4(22 \mathrm{~K})$ \\
\hline
\end{tabular}

${ }^{a}$ Values determined from the magnetic measurement.

${ }^{b}$ Values determined from the magnetic torque measurement. 
yielded to be $\xi_{a b}(0) \sim 10 \mathrm{~nm}$ and $\xi_{c}(0) \sim 3 \mathrm{~nm}$. These values are similar to the previous results obtained from magnetic measurements on powder samples ${ }^{27}$ or on single crystals $^{19-23}$ and the values determined from the calculation using the two band model, ${ }^{28}$ but do not agree with the reported $\gamma$-values determined from resistivity measurements on bulk samples ${ }^{15-18,10,11,29}$ and $c$-axis-oriented films, ${ }^{12-14}$ which are around 1.1 to 3 , as shown in Table II.

\section{CONCLUSION}

We have succeeded in synthesizing the single crystals under ambient pressure. So far the single crystals were produced by using special high pressure facilities. The synthesized single crystals are found to be of good quality $(R$ $\left.=0.020, R_{w}=0.027\right)$ judging from the structural analysis standard.

We measured the magnetic properties of single crystalline $\mathrm{MgB}_{2}$, and found a marked anisotropy; $H_{c 2}^{a b}$ reveals a positive curvature while $H_{c 2}^{c}$ increases linearly in the temperature dependence. The anisotropy ratio $\gamma=H_{c 2}^{a b} / H_{c 2}^{c}$ of the upper critical field is shown to be increased from about 1 near $T_{c}$ to 4.0 at $25 \mathrm{~K}$.

\section{ACKNOWLEDGMENTS}

We would like to thank K. Yamawaki, K. Noda, K. Ishikawa, H. Saito, Y. Takano, and K. Ohshima for useful discussions. This work has been supported in part by the $21 \mathrm{st}$ century COE program under the Ministry of Education, Culture, Sports, Science and Technology.
*Corresponding author. FAX: +81-45-924-5339. Email address: machida@lipro.msl.titech.ac.jp

${ }^{1}$ J. Nagamatsu, N. Nakagawa, T. Muranaka, Y. Zenitani, and J. Akimitsu, Nature (London) 410, 63 (2001).

${ }^{2}$ S. L. Bud'ko, G. Lapertot, C. Petrovic, C. E. Cunningham, N. Anderson, and P. C. Canfield, Phys. Rev. Lett. 86, 1877 (2001).

${ }^{3}$ J. W. Quilty, A. Yamamoto, and S. Tajima, Phys. Rev. Lett. 88, 087001 (2002).

${ }^{4}$ J. Kortus, I. I. Marzin, K. D. Belashchenko, V. P. Antropov, and L. L. Boyer, Phys. Rev. Lett. 86, 4656 (2001).

${ }^{5}$ A. Y. Liu, I. I. Marzin, and J. Kortus, Phys. Rev. Lett. 87, 087005 (2001)

${ }^{6}$ F. Bouquet, R. A. Fisher, N. E. Phillips, D. G. Hinks, and J. D. Jorgensen, Phys. Rev. Lett. 87, 047001 (2001).

${ }^{7}$ P. Szabó, P. Samuely, J. Kačmarčík, T. Klein, J. Marcus, D. Fruchart, and S. Miraglia, Phys. Rev. Lett. 87, 137005 (2001).

${ }^{8}$ F. Giubileo, D. Roditchev, W. Sacks, R. Lamy, D. X. Thanh, and J. Klein, Phys. Rev. Lett. 87, 177008 (2001).

${ }^{9}$ M. Iavarone, G. Karapetrov, A. E. Koshelev, W. K. Kwok, G. W. Crabtree, and D. G. Hinks, cond-mat/0203329 (unpublished).

${ }^{10}$ F. Simon et al., Phys. Rev. Lett. 87, 047002 (2001).

${ }^{11}$ O. F. de Lima, R. A. Ribeiro, M. A. Avila, C. A. Cardoso, and A. A. Coelho, Phys. Rev. Lett. 86, 5974 (2001).

${ }^{12}$ S. Patnaik et al., Supercond. Sci. Technol. 14, 315 (2001).

${ }^{13}$ M. H. Jung, M. Jaime, A. H. Lacerda, G. S. Boebinger, W. N. Kang, H. J. Kim, E. M. Choi, and S. I. Lee, Chem. Phys. Lett. 343, 447 (2001).

${ }^{14}$ R. J. Olsson et al., cond-mat/0201022 (unpublished).

${ }^{15}$ S. Lee, H. Mori, T. Masui, Y. Eltsev, A. Yamamota, and S. Tajima,
J. Phys. Soc. Jpn. 70, 2255 (2001).

${ }^{16}$ A. K. Pradhan, Z. X. Shi, M. Tokunaga, T. Tamegai, Y. Takano, K. Togano, H. Kito, and H. Ihara, Phys. Rev. B 64, 212509 (2001).

${ }^{17}$ K. H. P. Kim et al., Phys. Rev. B 65, 100510 (2002).

${ }^{18}$ M. Xu, H. Kitazawa, Y. Takano, J. Ye, K. Nishida, H. Abe, A. Matsushita, N. Tsujii, and G. Kido, Appl. Phys. Lett. 79, 2779 (2001).

${ }^{19}$ M. Angst, R. Puzniak, A. Wisniewski, J. Jun, S. M. Kazakov, J. Karpinski, J. Roos, and H. Keller, Phys. Rev. Lett. 88, 167004 (2001).

${ }^{20}$ U. Welp et al., cond-mat/0203337 (unpublished).

${ }^{21}$ M. Zehetmayer, M. Eisterer, H. W. Weber, J. Jun, S. M. Kazakov, J. Karpinski, and A. Wisniewski, cond-mat/0204199 (unpublished).

${ }^{22}$ A. V. Sologubenko, J. Jun, S. M. Kazakov, J. Karpinski, and H. R. Ott, Phys. Rev. B 65, 180505 (2002).

${ }^{23}$ L. Lyard et al., cond-mat/0206231 (unpublished).

${ }^{24}$ J. Karpinski et al., cond-mat/0207263 (unpublished).

${ }^{25}$ Y. Eltsev, S. Lee, K. Nakao, N. Chikumoto, S. Tajima, N. Koshizuka, and M. Murakami, cond-mat/0202133 (unpublished).

${ }^{26}$ K. Takanaka, J. Phys. Soc. Jpn. 52, 2173 (1983).

${ }^{27}$ S. L. Bud'ko and P. C. Canfield, cond-mat/0201085 (unpublished).

${ }^{28}$ P. Miranović, K. Machida, and V. G. Kogan, cond-mat/0206231 (unpublished).

${ }^{29}$ G. K. Perkins, J. Moore, Y. Bugoslavsky, L. F. Cohen, J. Jun, S. M. Kazakov, J. Karpinski, and A. D. Caplin, cond-mat/0205036 (unpublished). 